\title{
PIXEL-BASED LAND COVER CLASSIFICATION BY FUSING HYPERSPECTRAL AND LIDAR DATA
}

\author{
Farah Jahan, Mohammad Awrangjeb \\ Institute for Integrated and Intelligent Systems, Griffith University, Brisbane, Australia \\ Email: farah.jahan@griffithuni.edu.au,m.awrangjeb@griffith.edu.au
}

KEY WORDS: Hyperspectral, LiDAR, land-cover classification, feature combination, dimensionality reduction

\begin{abstract}
:
Land cover classification has many applications like forest management, urban planning, land use change identification and environment change analysis. The passive sensing of hyperspectral systems can be effective in describing the phenomenology of the observed area over hundreds of (narrow) spectral bands. On the other hand, the active sensing of LiDAR (Light Detection and Ranging) systems can be exploited for characterising topographical information of the area. As a result, the joint use of hyperspectral and LiDAR data provides a source of complementary information, which can greatly assist in the classification of complex classes. In this study, we fuse hyperspectral and LiDAR data for land cover classification. We do a pixel-wise classification on a disjoint set of training and testing samples for five different classes. We propose a new feature combination by fusing features from both hyperspectral and LiDAR, which achieves competent classification accuracy with low feature dimension, while the existing method requires high dimensional feature vector to achieve similar classification result. Also, for the reduction of the dimension of the feature vector, Principal Component Analysis (PCA) is used as it captures the variance of the samples with a limited number of Principal Components (PCs). We tested our classification method using PCA applied on hyperspectral bands only and combined hyperspectral and LiDAR features. Classification with support vector machine (SVM) and decision tree shows that our feature combination achieves better classification accuracy compared to the existing feature combination, while keeping the similar number of PCs. The experimental results also show that decision tree performs better than SVM and requires less execution time.
\end{abstract}

\section{INTRODUCTION}

The use of hyperspectral and Light detection and ranging (LiDAR) data for land cover classification and tree species classification has been an active topic of research in recent years (Dalponte et al., 2008; Matsuki et al., 2015; Man et al., 2014; Ghamisi et al., 2015). Hyperspectral sensors capture hundreds of narrow bands of the electromagnetic spectrum from visible to short-wave infrared wavelengths and provide detailed and continuous spectral information of different objects. On the other hand, LiDAR has been known as a vital method for characterizing vertical structures, including height and volume. As a result, the joint use of hyperspectral and LiDAR data provides a source of complementary information, which can greatly assist in the classification of complex land covers.

Land cover classification methods using the fusion of hyperspectral and LiDAR data can be classified into three categories: pixellevel, feature-level and decision level fusion (Priem and Canters, 2016; Man et al., 2014). Pixel-level fusion is the lowest level of image fusion which provides original information but is vulnerable to noise and requires relatively long processing time. Feature-level fusion extracts spatial features using object shape and neighbourhood information, spectral and topographical features by keeping sufficient information with a certain level of accuracy. Decision level fusion is the highest level of fusion which combines the classification results in classifier level.

Luo et al. (2016) performed land cover classification using hyperspectral and LiDAR data and classified seven different classes including buildings, road, water bodies, forests, grassland, cropland and barren land. They applied pixel based fusion (also called layer stacking) and Principle Component Analysis (PCA) on fused hyperspectral and LiDAR features. The fusion was done on varying spatial resolutions, which helped to select the best performing spatial resolution. In this framework, PCA was applied on combined hyperspectral and LiDAR features cube ( all features from both modalities applied on a cube), which was different from most of the related work such as Khodadadzadeh et al. (2015) where PCA was used on a single modality (hyperspectral). Man et al. (2015) fused hyperspectral and LiDAR data for urban area classification. Firstly, they used pixel-based fusion and classified land covers separately using support vector machine (SVM) and maximum likelihood (MLC) classifiers. To improve the accuracy they combined pixel and object-based classification. In the object-based classification, they set some rules depending on the shape and nature of the classes which were applicable for the dataset that they used but it was not generalised for all the urban areas. Morchhale et al. (2016) investigated classification from the pixel-level fusion of hyperspectral and LiDAR data by applying convolutional neural networks (CNN). Their experimental results proved that pixel-level fusion is an effective approach for the classification using CNN. However, they ignored natural spatial relationship among the nearby pixels.

Ghamisi et al. (2015) proposed an automatic method for fusing hyperspectral and LiDAR data. They used attribute profile (AP) for capturing spatial information both from hyperspectral and LiDAR. For extracting spectral information from hyperspectral data three different supervised feature extraction techniques were used. All the extracted features from hyperspectral and LiDAR were fused into a stacked vector and classified by supervised SVM and Random tree classifiers. Supervised feature extraction step was unnecessary for the used data sets because it increased the complexity of the classifiers without making significant improvements. The winning algorithm (Debes et al., 2014) 
of 2013 IEEE GRSS Data fusion contest introduced a parallel unsupervised and supervised classification. Wang and Glennie (2015) used feature level fusion of synthesised waveform (SWF) and hyperspectral data for land-cover classification. Recently, the fusion of multiple classifiers also improved the classification accuracy which is a form of decision level fusion (Bigdeli et al., 2015).

From the above discussion, it is clear that most of the contributions of the previous works were based on pixel-level after extracting spectral and topological features from hyperspectral and LiDAR data. To reduce the misclassification, spatial relationships among the pixels and the shape and volume of the object were also considered after the pixel-based classification as a post classification step. It is a great challenge to select a reduced number of discriminative features from pixel level and selection of appropriate classifier/classifiers combination which give a generalised model with good performance.

In our method, we undertake pixel-based classification approach using the features extracted from hyperspectral and LiDAR data. Our extracted feature combination is able to classify five different classes with a high classification accuracy with the dimensionality of 8 . For the classification, we used supervised classifier SVM and decision tree. SVM is the most popular supervised classifier for the classification of hyperspectral and LiDAR data (Dalponte et al., 2008; Luo et al., 2016; Gu et al., 2015; Wang and Glennie, 2015). However, in our case, the decision tree is performing better than SVM. The main aim of our study is to examine the performance of different features from two modalities (hyperspectral and LiDAR) and fuse them in different ways to improve the classification accuracies of various land-cover classes. To accomplish this goal our study contributes in the following ways:

- We implement an image inpainting algorithm for replacing missing LiDAR points for improving the quality of Digital Surface Model (DSM) and intensity images from LiDAR point cloud.

- We compare the performance of our feature combination with the feature combination proposed in (Luo et al., 2016). Our proposed additional features with the features used by Luo et al. (2016) improves the classification accuracies. Additionally, one of our feature combination without using all hyperspectral bands provides impressive classification accuracies with limited feature vector dimensionality of 8 .

- We implement two fusion techniques named layer stacking and Principal Component Analysis (PCA). We use PCA in two different ways. Firstly, PCA is applied on hyperspectral bands only and additional features with the first few PCs were added. Secondly, PCA is applied on the whole, feature vector from hyperspectral and LiDAR as Luo et al. (2016). Our former technique for using PCA provides higher classification accuracies. Also, we measure the classification accuracies of our feature combination and the feature combination proposed by Luo et al. (2016) when applying PCA on the whole feature vector. Our feature combination achieves higher classification accuracies with the same number of PCs than the mentioned existing one using the decision tree.

- Our method for classifying land cover classes is not dependent on any prior knowledge like road width/tree height. It can be used in other datasets without any adjustment that is required by some existing method (Man et al., 2015).
- Most of the recent land-cover classification used SVM for classification and SVM outperformed other classifiers. In our case, decision tree outperforms SVM in most of the feature combination with a limited number of features. Because our selected features easily categorise samples which fit with the construction structure of the decision tree. As a result, using decision tree we achieve good classification accuracies with a limited number of features. Limited number of features reduce the dimension of the feature vector and simplifies the classification process.

- Our methods execute in reasonable CPU processing time. Comparing two supervised classifiers, decision tree outperforms SVM in terms of execution time. The reason behind faster classification by decision tree is that constructing decision tree is computationally inexpensive even when the size of the training set is large.

In this paper section 2 illustrates our proposed methodology where we explain all our approaches in detail. We discuss about registration process, data recovery, feature extraction and mapping in that section. Section 3 discusses our experimental setup, brief introduction of input data and experimental outcomes. We conclude novelty of our works and future plans in section 4 .

\section{METHODOLOGY}

Our framework consists of three main sections including preprocessing and feature extraction, fusion and classification, same as other research done on land cover classification. After extracting features from both hyperspectral and LiDAR data separately, we fuse them for the classification of five different land cover classes, i.e., "Road", "Tree", "Grass", "Water" and "Soil". Figure 1 shows the framework of our land-cover classification system.

\subsection{Data Preprocessing}

In Figure 1, the first section of preprocessing shows the steps of processing and feature extraction both from hyperspectral and $\mathrm{Li}$ DAR data. It shows two flows of steps coming from LiDAR and hyperspectral that are combined in the co-registration step. In the flow coming from LiDAR, LiDAR point clouds are initially rasterized according to the pixel size of hyperspectral. In our case, it is 0.5 centimetre. Digital Elevation Model (DEM) is created from the ground return of LiDAR data using ENVI 5.3. The first pulse Digital Surface Model (FP_DSM) is generated through rasterizing the first LiDAR return of pixel locations. In the same way, the last pulse Digital Surface Model (LP_DSM) is created. We generate the intensity image by calculating the intensity of the first pulse.

Figure 2(a) shows LiDAR point cloud aligned with the hyperspectral RGB image of an area. From the figure, we can see LiDAR points are missing in several regions. For recovering missing LiDAR points we use inpainting process by Pingel et al. (2013). The reason behind applying inpainting is, our missing LiDAR points are random across the whole image so simple interpolation or regression model is not able to produce the desired result. After creating FP_DSM, LP_DSM and intensity images, we apply inpainting process for generating missing point values, which improves the quality of FP_DSM, LP_DSM and intensity image to a great extent. The inpainting technique is based on least square approach, which is mathematically explained by 


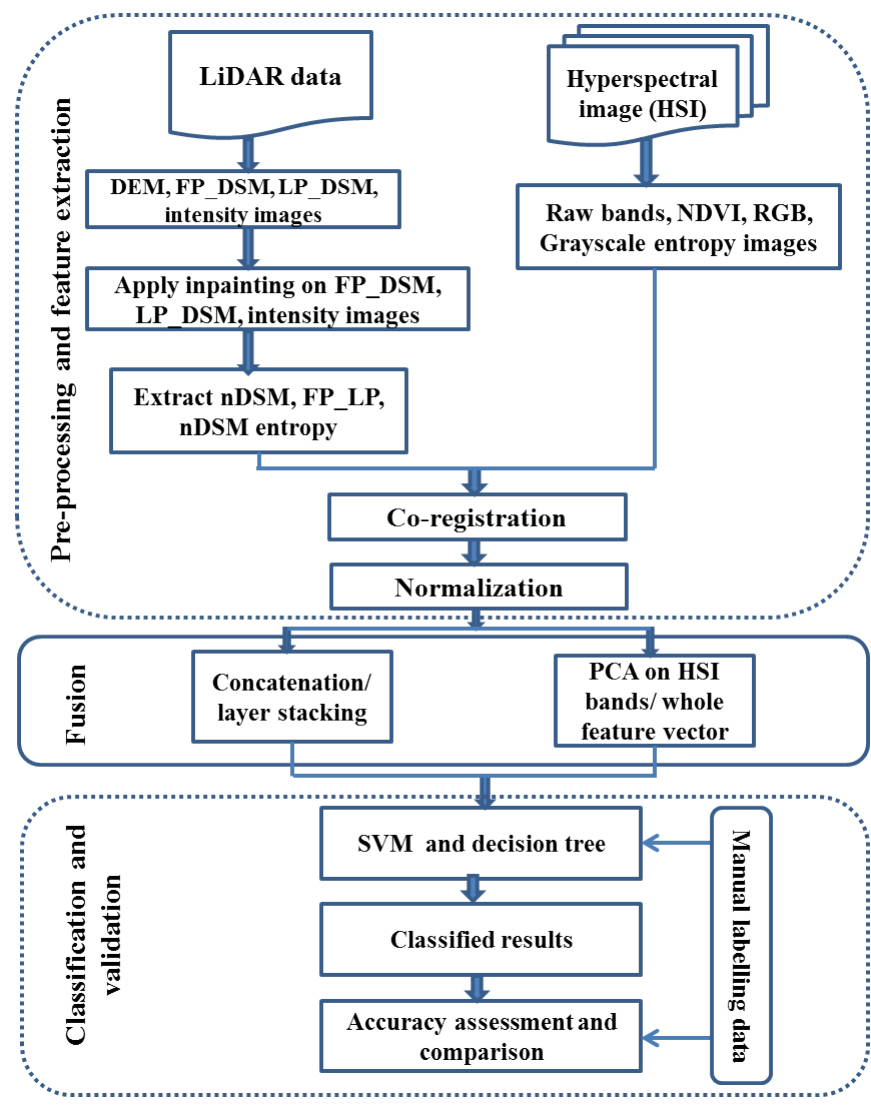

Figure 1. Proposed Framework.

function $S$ characterized by two unknown values $\beta_{1}$ and $\beta_{2}$ in Equations (1) and (2):

$\boldsymbol{S}\left(\boldsymbol{\beta}_{\mathbf{1}}, \boldsymbol{\beta}_{\mathbf{2}}\right)=\left[10-\boldsymbol{\beta}_{\mathbf{1}}\right]^{2}+\left[\boldsymbol{\beta}_{\mathbf{2}}-\boldsymbol{\beta}_{\mathbf{1}}\right]^{2}+\left[3-\boldsymbol{\beta}_{\mathbf{1}}\right]^{2}+\left[5-\boldsymbol{\beta}_{\mathbf{1}}\right]^{2}$

We will get the unknown values $\beta_{1}$ and $\beta_{2}$ by solving the partial derivative equations of the following:

$$
\begin{aligned}
& \frac{\partial S}{\partial \boldsymbol{\beta}_{\mathbf{1}}}=0 \\
& \frac{\partial S}{\partial \boldsymbol{\beta}_{\mathbf{2}}}=0
\end{aligned}
$$

After co-registration of hyperspectral and LiDAR point cloud, we are able to generate a feature vector for each pixel using features from both hyperspectral and LiDAR. From Figure 2(a) it is observed that after registration LiDAR point cloud is not properly aligned with the hyperspectral image. From the image, it is clear that LiDAR point cloud is shifted upward in the direction of Yaxis. We manually corrected this registration error for the proper alignment of these two types of data. Figure 2(b) and Figure 2(c) show the DSM with missing LiDAR points (white colours) and the processed DSM after applying inpainting. We normalise the feature vector of each pixel between 0 and 1 as expressed in Equation (3). In Equation (3), $x=\left(x_{1}, x_{2}, \ldots, x_{n}\right)$ and $z_{i}$ is the $i^{\text {th }}$ normalized data. We do this normalisation because different features have different ranges of values. But when we fuse them for classification, different ranges of values disrupt the learning and classifying processes of the classifier.

$$
z_{i}=\frac{x_{i}-\min (x)}{\max (x)-\min (x)}
$$

Figure 3 shows the RGB images of five different areas from where

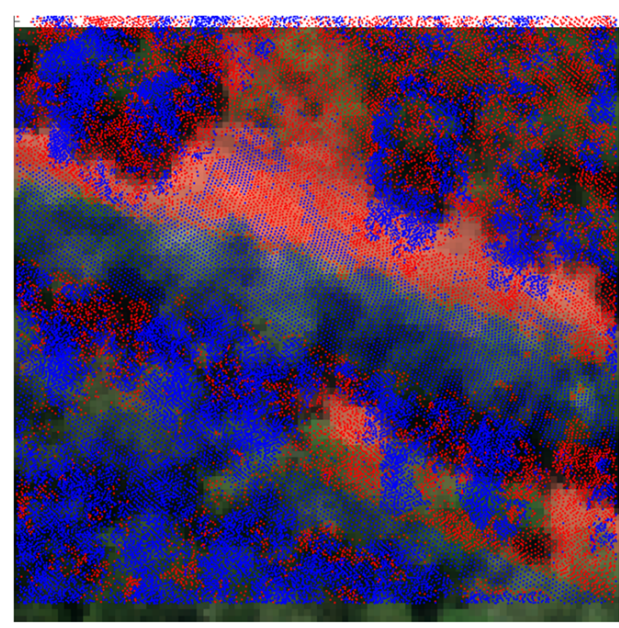

(a) LiDAR point cloud aligned with hyperspectral RGB of Area 1 $(94 \times 98)$

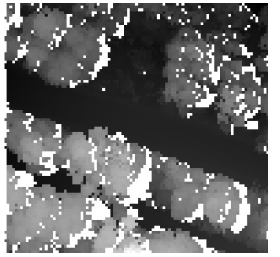

(b) Area 1 DSM with missing points $(334 \times 479)$

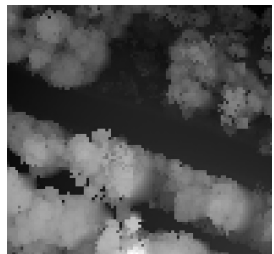

(c) Area 1 DSM after applying Inpainting
Figure 2. Registration error and inpainting algorithm for recovering missing LiDAR points.

we collected samples for our experiment for the classification of five different land cover classes.

\subsection{Feature Extraction}

Feature extraction is an important step for land-cover classification. The classification accuracy depends on the discriminative property of the features extracted from various classes. In this research, we extract spectral and texture features from the hyperspectral image (HSI). From LiDAR, we extract various height information such as DEM (Digital Elevation Model), DSM (Digital Surface Model), nDSM (Normalised Digital Surface Model), difference between the first and the last LiDAR returns (FP_LP), the intensity of the LiDAR pulse and texture information of $\mathrm{nDSM}$ like entropy. The following subsection discusses the texture and colour features we use in the land cover classification.

- Normalised Difference Vegetation Index (NDVI) The Normalised Difference Vegetation Index (NDVI) is a numerical indicator to evaluate whether the target being observed contains live green vegetation Holm AM (1987).

In our case, we calculate NDVI using the following equation

$$
N D V I=\frac{R_{N I R}-R_{r e d}}{R_{N I R}+R_{\text {red }}}
$$

We use NIR (795.16nm, band 42) and Red (679.46nm, band 30) for calculating NDVI using Equation (4). Figure 4(h) shows NDVI image of an area. The tree and grass areas are nearly white for high NDVI. On the other hand, road/soil areas are black for low NDVI.

- Digital Elevation Model (DEM) Digital Elevation Model 
(DEM) is typically used to represent the height of the bareearth terrain. Figure 4(a) shows the DEM of Area 1.

- Digital Surface Model (DSM) Digital Surface Model (DSM) captures the height of natural and built features on earth.

In this study, we implement image inpainting techniques for filling missing points values of LiDAR return. Figure 4(b) shows the DSM of Area 1. We discussed our inpainting techniques in the previous section - data preprocessing.

- Normalized Digital Surface Model (nDSM) Normalized Digital Surface Model (nDSM) is calculated by subtracting bare earth returns from the first return reflected by an object on the ground. Figure 4(c) shows the DSM of Area 1.

- Difference Between the First and Last LiDAR Returns (FP_LP) In modern LiDAR systems, multiple returns are received for a single laser pulse. In the case of a tree, the laser pulse may go down and partially reflect from different parts of the tree leaves, trunk and branches until it finally hits the bare ground. If there is a solid object like a building or ground, it will just hit the surface. The difference between the first and the last LiDAR returns represents an important property of the reflecting object. We measure the height of first and last LiDAR return of each pixel location $(i, j)$ and calculate the difference between them. In the case of a tree, the difference is larger than a road/bare surface. Figure 4(e) represents the difference between the first and the last LiDAR returns of a land cover area.

- Intensity LiDAR sensor measures the relative strength of the return pulse which is called intensity. Figure 4(d) shows the intensity image of Area 1. From Figure 4(d) we can observe that the intensity of grass and tree regions varies frequently but the intensity of road is quite stable.

- Entropy In our land cover classification, entropy gives important texture information for the classification. For example, the surface of road, tree, grass, water and soil differ from one another in terms of texture smoothness. The surface texture of tree and grass are rougher when we compare these to road and water.

Our hyperspectral images contain 62 bands. We select 3 bands associated with red (wavelength 650.84, band 27), green (wavelength 536.64, band 15) and blue (wavelength 564.92, band 18) to generate an RGB image from the hyperspectral image. The grayscale image is obtained from the RGB image as follows Gonzalez et al. (2003). After converting the RGB image into the gray-scale image we calculate the entropy of the gray-scale image.

We also calculate entropy from the $n D S M$ (Normalised Dig ital Surface Model). Trees give higher entropy in $n D S M$ as the surface of the trees frequently varies with height but the road gives lower nDSM entropy. Figure 4(g) represents the gray-level entropy of Area 3 and 4(f) shows the nDSM entropy of the same Area 2. For gray-level entropy, water represents low entropy value for its smooth texture but grass, tree and soil are contain higher entropy values for their rough texture.

\subsection{Data fusion of extracted features}

After extracting the features from every pixel, features are concatenated to produce the signature of each pixel. We produce
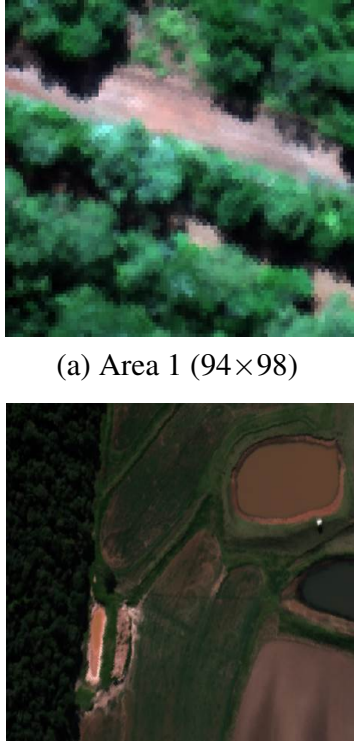

(c) Area $3(334 \times 479)$ (a) Area $1(94 \times 98)$

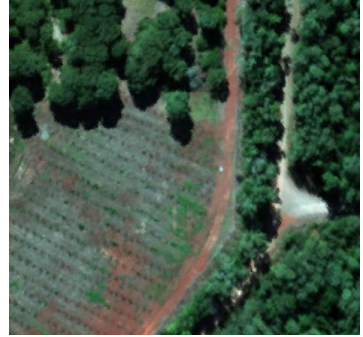

(b) Area $2(234 \times 272)$

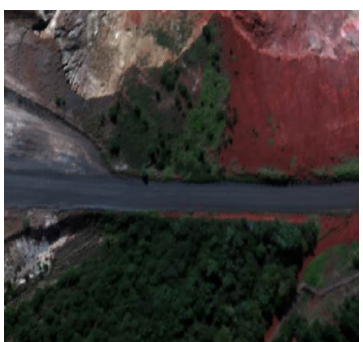

(d) Area $4(255 \times 368)$

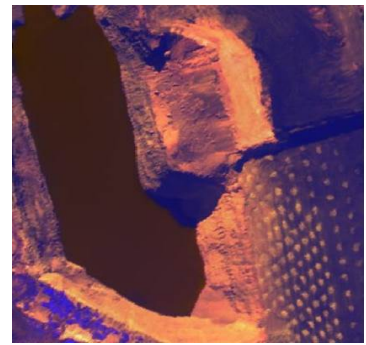

(e) Area $5(334 \times 392)$

Figure 3. RGB images and dimensions of five different areas.

nine different types of signatures by combining different features. For fusing data from hyperspectral and LiDAR, we applied two strategies. One is simple layer stacking/ concatenation and another is Principal Component Analysis (PCA).

- Concatenation/Layer stacking This is commonly used method for the fusion of hyperspectral and LiDAR. In layer stacking, different features are linearly concatenated to produce the signature of each pixel.

- Principal Component Analysis (PCA) PCA is a useful statistical technique for finding patterns in data of high dimension. PCA transforms the data into a lower dimensional subspace which is optimal in terms of sum-of-squared error (Jolliffe, 2002). PCA reduces the dimensionality of data into a new set of uncorrelated variables, called Principal components (PCs), by a linear transformation of the input data. The first PC has the largest variance (largest eigenvalue), the second component has the second largest variance (second largest eigenvalue), etc. PCs are orthogonal to each other and are ordered according to descending eigenvalues. We use PCA in two different ways in the fusion technique as discussed before. All the classification results will be shown in the results and discussion section.

\subsection{Classification}

Classification is a pervasive problem that encompasses many diverse applications. From the machine learning point of view, machines are primarily trained by known examples according to the 


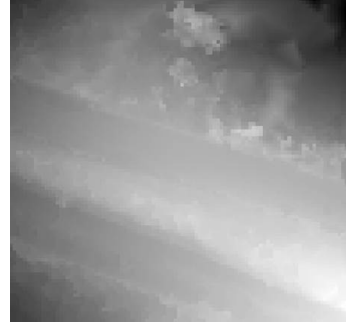

(a) DEM of Area 1

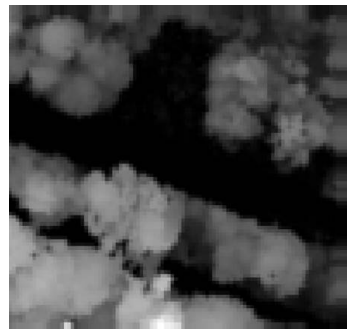

(c) nDSM of Area 1

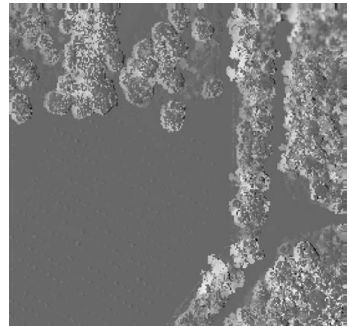

(e) FP_LP of Area 2

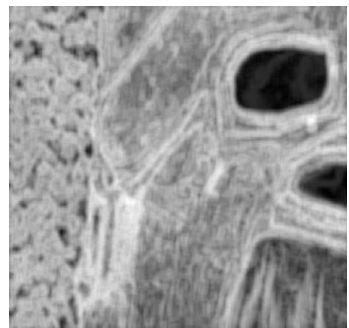

(g) Gray Entropy of Area 3

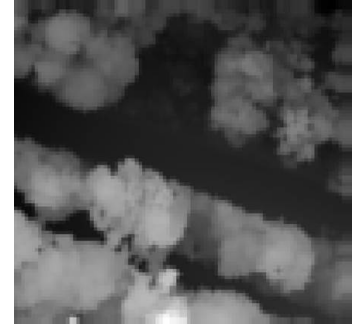

(b) DSM of Area 1

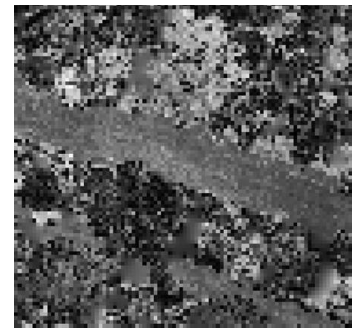

(d) Intensity of Area 1

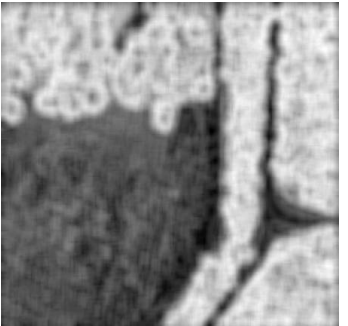

(f) nDSM entropy of Area 2

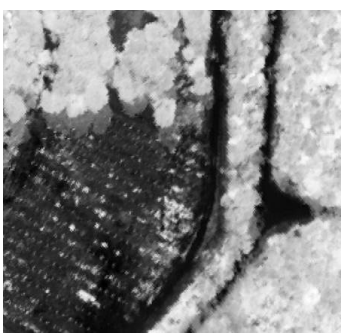

(h) NDVI of Area 2
Figure 4. Eight different features extracted from each area

nature of classification we want to apply. After training, a knowledge/model is created by which the machine can categorise unknown samples into several classes based on their attribute values. SVM is the most popular classifier used to classify fused hyperspectral and LiDAR data and in most of the cases, it outperforms Random forests and Maximum likelihood classifiers. For our land cover classification, we use supervised classifiers: Linear SVM and decision tree. The classification accuracies and execution time are reported in the results and discussion section. Decision tree achieved higher classification accuracies than SVM for our dataset and the feature combinations we used for classification. Also, decision tree classifies data much faster than SVM. The reason behind the smaller execution time of decision tree is that SVM requires parameter tuning to achieve optimal results, while decision tree does not require such tuning process.

\section{EXPERIMENTAL RESULT}

The data was collected from "Yarraman State Forest" and its adjacent area located in $170 \mathrm{~km}$ north-west of Brisbane, Queensland,
Australia. The total area was almost $8 \mathrm{~km}^{2}$. The data was captured 2015 between the month June to July. Table 1 and Table 2 show the sensor parameters which captured LiDAR and hyperspectral data.

We manually labelled pixels with the help of Google maps. We collected our samples from five different areas shown in Figure 3. Table 3 shows the number of pixels for five different classes. We create 10 different training and testing sets by randomly splitting pixels equally from each class for the evaluation of our methods.

Table 1. LiDAR data

\begin{tabular}{|l|l|}
\hline Parameter & Specification \\
\hline Recorded returns & 6 \\
\hline Average point spacing & $0.2 \mathrm{~m}$ \\
\hline
\end{tabular}

Table 2. Hyperspectral data

\begin{tabular}{|l|l|}
\hline Parameter & Specification \\
\hline Sensor name & AISA \\
\hline Number of Bands & $62(408.54 \mathrm{~nm}-990.62 \mathrm{~nm})$ \\
\hline Spatial resolution & $0.5 \mathrm{~m}$ \\
\hline Spectral resolution & $8.94-9.81 \mathrm{~nm}$ \\
\hline
\end{tabular}

To compare the performance of our features, we develop nine methods by exploring different combinations of the features. We compare the performance of our method with other approaches. We briefly describe our methods as follows:

- Method 1: All 62 bands from hyperspectral data are used for the classification.

- Method 2: Only LiDAR DSM is used for the classification.

- Method 3: All hyperspectral bands and LiDAR DSM, DTM, $\mathrm{nDSM}$ and intensity are used for the classification (Luo et al., 2016).

- Method 4: All hyperspectral bands, Gray entropy, NDVI and LiDAR DSM, DTM, nDSM, intensity, FP_LP, nDSM entropy are used for the classification.

- Method 5: Gray entropy, NDVI from hyperspectral and LiDAR DSM, DTM, nDSM, Intensity, FP_LP, nDSM entropy are used for the classification.

- Method 6: PCA is used to reduce the dimensionality of HSI data. PCs, LiDAR DSM, DTM, nDSM, Intensity are used for the classification.

- Method 7: PCA is used to reduce the dimensionality of HSI data. PCs, Gray entropy, NDVI, LiDAR DSM, DTM, nDSM, Intensity, FP_LP, nDSM entropy are used for the classification.

- Method 8: PCA is applied on the feature vector used in Method 3 (Luo et al., 2016). The PCs are used for the classification.

- Method 9: PCA is applied on the feature vector used in Method 4. The PCs are used for the classification.

Table 4 shows the features and their relationship with nine different methods. Table 5 shows the classification accuracies and execution time by each method by using SVM and decision tree. We 
Table 3. Distribution of samples collected from five areas for five different classes.

\begin{tabular}{|c|c|}
\hline Class & Number of Pixels \\
\hline Road & 845 \\
\hline Tree & 1422 \\
\hline Grass & 821 \\
\hline Water & 409 \\
\hline Ground & 810 \\
\hline Total & 4307 \\
\hline
\end{tabular}

also graphically explain the performance of 9 different methods shows in Figure 5. Method 6 to 9 which use PCA, we consider the accuracies of 5 PCs in Table 5 and Figure 5 but we recorded accuracies of all PCs. The graphs which shows the accuracies related to each PCs are shown in Figure 6 and Figure 7. All the methods were programmed in MATLAB on a computer having an Intel Core (TM) i5-4590 processor $(3.30 \mathrm{GHz})$ and $8 \mathrm{~GB}$ memory.

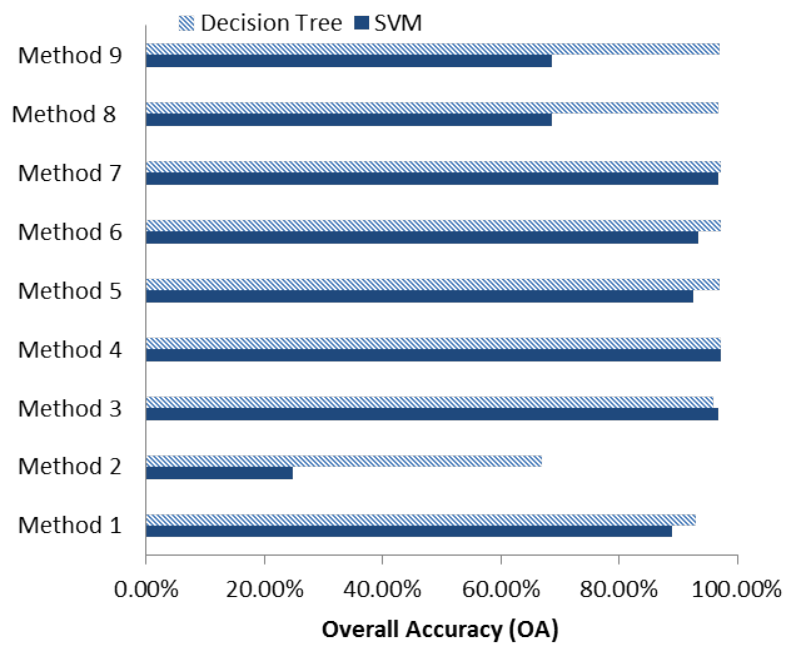

Figure 5. Performance of different methods using SVM and decision tree.

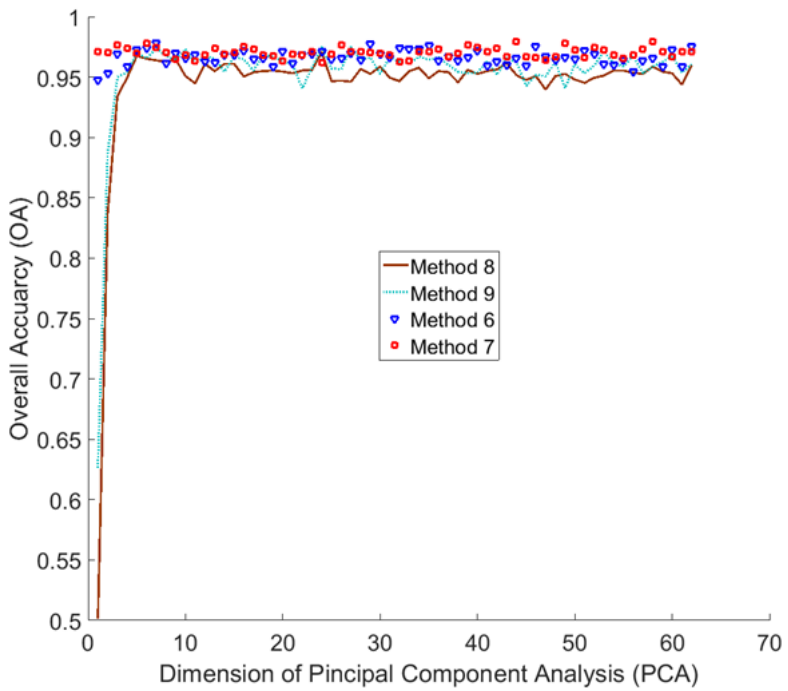

Figure 6. Dimension of PCA and Overall Accuracy (OA) obtained by decision tree.

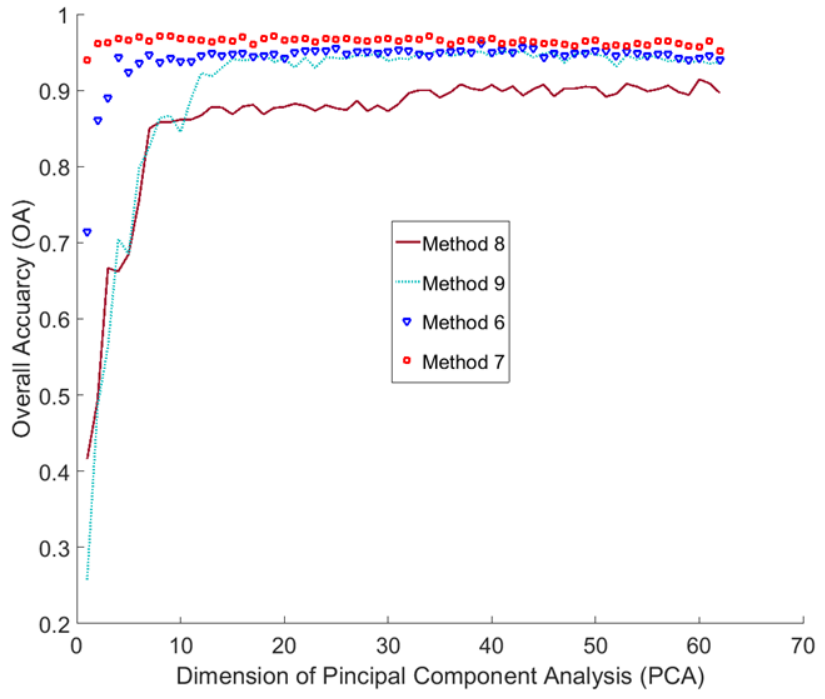

Figure 7. Dimension of PCA and Overall Accuracy (OA) obtained by SVM.

\subsection{Algorithm Evaluation}

Accuracy assessment is based on confusion matrix generated by Matlab R2016a. The confusion matrix is $n \times n$ matrix where $n$ is the number of classes. In the confusion matrix, each row represents the actual class/ground truth and each column represents the predicted class. From the confusion matrix, we calculated overall accuracy (OA) and average accuracy (AA). Before that, we calculated precision and class-wise accuracy/recall. The Equations are as follows:

$$
\begin{gathered}
\text { Precision }=\frac{\text { True positive }}{\text { True positive }+ \text { False positive }} \\
\text { Accuracy }=\frac{\text { True positive }}{\text { True positive }+ \text { False Negative }} \\
\text { OverallAccuracy }=\frac{\sum \text { Correctly Classified Samples }}{\text { Total Number of Samples }} \\
\text { AverageAccuracy }=\frac{\sum \text { Acuuracy of all classes }}{\text { Number of classes }}
\end{gathered}
$$

\subsection{Results and Discussion}

Table 5 provides information related to the classification accuracies of SVM and decision tree of nine different methods. For Method 1, raw 62 hyperspectral bands were used as features so the dimension of the feature vector was 62. Decision tree obtained higher classification accuracy than SVM which were $4.72 \%$ higher for AA and $4.05 \%$ higher for OA. Method 2 used LiDAR DSM only as a feature. Decision tree improved OA by $42.13 \%$ than SVM. For Method 3, the AA and OA we slightly improved by the SVM than decision tree which were $0.74 \%$ and $0.86 \%$, respectively. Like Method 3, in Method 4 SVM AA and OA were a bit higher than decision tree, which were $0.10 \%$ and $0.50 \%$. Method 5 used extracted features from hyperspectral and LiDAR without considering raw hyperspectral bands with dimensionality 8 , where decision tree was performing better by improving AA by $4.5 \%$ and OA by $3.85 \%$ than SVM. Both Method 6 and Method 7 reduced hyperspectral bands using PCA and concatenated it with additional features from LiDAR and hyperspectral. In Method 6, we considered five PCs from hyperspectral bands and concate- 
Table 4. Relationship between features and different methods. ' $\mathrm{Y}$ ' means that a particular feature is used by a method.

\begin{tabular}{|c|c|c|c|c|c|c|c|c|c|c|}
\hline Sl.no & Features & $\begin{array}{c}\text { Method } \\
1\end{array}$ & $\begin{array}{c}\text { Method } \\
2\end{array}$ & $\begin{array}{c}\text { Method } \\
3\end{array}$ & $\begin{array}{c}\text { Method } \\
4\end{array}$ & $\begin{array}{l}\text { Method } \\
5\end{array}$ & $\begin{array}{l}\text { Method } \\
6\end{array}$ & $\begin{array}{c}\text { Method } \\
7\end{array}$ & $\begin{array}{l}\text { Method } \\
8\end{array}$ & $\begin{array}{l}\text { Method } \\
9\end{array}$ \\
\hline 1 & DSM & & $\mathrm{Y}$ & Y & $\mathrm{Y}$ & $\mathrm{Y}$ & $\mathrm{Y}$ & $\mathrm{Y}$ & & \\
\hline 2 & DTM & & & $\mathrm{Y}$ & $\mathrm{Y}$ & $\mathrm{Y}$ & $\mathrm{Y}$ & $\mathrm{Y}$ & & \\
\hline 3 & nDSM & & & $\mathrm{Y}$ & $\mathrm{Y}$ & $\mathrm{Y}$ & $\mathrm{Y}$ & $\mathrm{Y}$ & & \\
\hline 4 & Intensity & & & $\mathrm{Y}$ & $\mathrm{Y}$ & $\mathrm{Y}$ & $\mathrm{Y}$ & $\mathrm{Y}$ & & \\
\hline 5 & FP LP & & & & $\mathrm{Y}$ & $\mathrm{Y}$ & & $\mathrm{Y}$ & & \\
\hline 6 & nDSM Entropy & & & & $\mathrm{Y}$ & $\mathrm{Y}$ & & $\mathrm{Y}$ & & \\
\hline 7 & Gray Entropy & & & & $\mathrm{Y}$ & $\mathrm{Y}$ & & $\mathrm{Y}$ & & \\
\hline 8 & NDVI & & & & $\mathrm{Y}$ & $\mathrm{Y}$ & & $\mathrm{Y}$ & & \\
\hline 9 & HSI bands & $\mathrm{Y}$ & & $\mathrm{Y}$ & $\mathrm{Y}$ & & & & & \\
\hline 10 & $\begin{array}{c}\text { PCA } \\
(\text { Sl no.9) }\end{array}$ & & & & & & $\mathrm{Y}$ & $\mathrm{Y}$ & & \\
\hline 11 & $\begin{array}{c}\text { PCA } \\
\text { (Sl no. } 1 \text { to } 4 \text { and 9) }\end{array}$ & & & & & & & & Y & \\
\hline 12 & $\begin{array}{c}\text { PCA } \\
\text { (Sl no. } 1 \text { to } 9)\end{array}$ & & & & & & & & & $\mathrm{Y}$ \\
\hline
\end{tabular}

Table 5. Classification accuracies from SVM and decision tree.

\begin{tabular}{|l|l|l|l|l|l|l|l|l|l|l|l|}
\hline Features & Dimension & \multicolumn{4}{|c|}{ SVM } & \multicolumn{5}{c|}{ Decision tree } \\
\hline & & $\begin{array}{l}\text { Mean } \\
(\mathrm{AA}) \\
(\%)\end{array}$ & $\begin{array}{l}\text { STD } \\
(\mathrm{AA}) \\
(\%)\end{array}$ & $\begin{array}{l}\text { Mean } \\
(\mathrm{OA}) \\
(\%)\end{array}$ & $\begin{array}{l}\text { STD } \\
(\mathrm{OA}) \\
(\%)\end{array}$ & $\begin{array}{l}\text { Time } \\
(\mathrm{sec})\end{array}$ & $\begin{array}{l}\text { Mean } \\
(\mathrm{AA}) \\
(\%)\end{array}$ & $\begin{array}{l}\text { STD } \\
(\mathrm{AA}) \\
(\%)\end{array}$ & $\begin{array}{l}\text { Mean } \\
(\mathrm{OA}) \\
(\%)\end{array}$ & $\begin{array}{l}\text { STD } \\
(\mathrm{OA}) \\
(\%)\end{array}$ & $\begin{array}{l}\text { Time } \\
(\mathrm{sec})\end{array}$ \\
\hline Method 1 & 62 & 88.86 & 6.92 & 88.89 & 6.83 & 71.98 & 93.58 & 0.71 & 92.94 & 0.73 & 5.10 \\
\hline Method 2 & 1 & 35.63 & 3.07 & 24.72 & 2.86 & 24.16 & 63.66 & 0.87 & 66.85 & 1.09 & 0.31 \\
\hline Method 3 & 66 & 96.90 & 0.19 & 96.79 & 0.15 & 50.76 & 96.16 & 0.53 & 95.93 & 0.54 & 0.62 \\
\hline Method 4 & 70 & 97.35 & 0.25 & 97.27 & 0.25 & 45.58 & 97.25 & 0.35 & 97.22 & 0.36 & 0.65 \\
\hline Method 5 & 8 & 92.37 & 0.51 & 92.47 & 0.47 & 27.45 & 97.06 & 0.25 & 97.06 & 0.22 & 0.22 \\
\hline Method 6 & 9 & 92.78 & 0.90 & 93.33 & 0.53 & 29.32 & 97.28 & 0.48 & 97.18 & 0.50 & 0.19 \\
\hline Method 7 & 13 & 96.72 & 0.25 & 96.76 & 0.23 & 30.09 & 97.22 & 0.73 & 97.20 & 0.71 & 0.23 \\
\hline Method 8 & 5 & 65.06 & 0.22 & 68.51 & 0.21 & 25.42 & 96.89 & 0.69 & 96.75 & 0.68 & 0.13 \\
\hline Method 9 & 5 & 59.56 & 0.23 & 68.51 & 0.22 & 24.41 & 97.05 & 0.82 & 96.98 & 0.71 & 0.15 \\
\hline
\end{tabular}

nate them with four LiDAR features DSM, DTM, nDSM and Intensity. Decision tree AA and OA were $4.5 \%$ and $3.85 \%$ higher than SVM, respectively. In Method 7, we added additional features FP_LP, nDSM_Entropy, Gray_Entropy and NDVI with the features of Method 6. The performance of SVM was improved for Method 7 than Method 6. As before, the performance of decision tree was a bit higher than SVM for Method 7. Methods 8 and 9 applied PCA on all the features coming from hyperspectral and LiDAR. The feature combination of Method 8 was similar to Method 3 while Method 9 was similar to Method 4. For Method 8 and Method 9, decision tree performed much higher than SVM, approximately $31.83 \%$ higher for AA and $28.24 \%$ higher for OA.

Method 1 to 5 were based on layer stacking feature fusion from hyperspectral and LiDAR. Among them, Method 4 delivered the highest OA and AA using both SVM and decision tree with a feature vector dimensionality 70 . Method 3 represented the feature combination used by (Luo et al., 2016) with dimensionality 66. If we compared the performance of Method 5 and Method 3 , Method 5 improved AA by $0.9 \%$ and OA by $1.13 \%$ using decision tree compared to Method 3 while reducing dimensionality from 66 to 8. If we compared the performance of Method 1 and Method 2 with Method 3, Method 4, Method 5, we also noticed that fusing features from hyperspectral and LiDAR improved the classification accuracies as well as reduced the feature vector dimensionality (Method 5) to a great extent.

Method 6 to Method 9 used PCA for feature fusion from hyper- spectral and LiDAR. In Method 6 and Method 7 we used PCA to reduce the bands of hyperspectral and add additional features from hyperspectral and LiDAR. Method 8 and Method 9 apply PCA on features from both hyperspectral and LiDAR as existing method (Luo et al., 2016). Method 6 and Method 8 use the feature combination proposed by (Luo et al., 2016). Table 5 and Figure 5 only considered the accuracies of 5 PCs. The graph in Figure 6 and Figure 7 recorded the accuracies of Method 6 to Method 9 considering all PCs. From Table 5 and figures it is clear that Method 6 and Method 7 perform better than Method 8 and Method 9. Keeping the same number of PCs our proposed feature combination Method 7 and Method 9 perform better than Method 6 and Method 8 using both decision tree and SVM.

\section{CONCLUSION}

In this paper, a novel combination of features both from hyperspectral and LiDAR is used for the classification. Based on the experimental results, several conclusions can be made such as FP_LP, nDSM_Entropy and Gray_Entropy help to discriminate pixels in addition to commonly used features like DSM, DTM, nDSM, Intensity and NDVI. Entropy from both hyperspectral and LiDAR gives us the spatial relationship among pixels. Fusing features from hyperspectral and LiDAR improves classification accuracies from $4.12 \%$ for decision tree and $3.58 \%$ for SVM by reducing feature dimension from 62 (all hyperspectral bands) to 8 (Method 5). Also, when we compare our feature combination 
(Method 5) with an existing one (Method 3), our proposed feature combination improves OA by $1.13 \%$ while reducing feature dimension from 66 to 8 . PCA applied on only HSI bands rather than HSI bands and other LiDAR features prove to be effective for the used dataset. Also, our feature combination (Method 9) achieves higher classification accuracies by using decision tree than the existing feature combination (Method 8) while keeping the same number of principal components (5). Decision tree achieves higher classification accuracies than SVM using a limited number of features (reduced feature dimension). On the other hand, SVM achi-eves better accuracies for a large number of features (Method 3 and 4). Our aim is to achieve good classification accuracy with a limited number of features that is ignored by other existing studies. Our experimental results proved that decision tree classifier achieves a better result with a limited number of features and also faster than SVM. Our selected feature combination is effective for the discriminative construction of decision tree from the training set, which is also generalised for various land cover classes.

In future, we will try to apply other feature reduction techniques and more advanced spatial feature extraction techniques. Besides this, we are trying to develop a novel feature fusion technique instead of layer stacking method.

\section{REFERENCES}

Bigdeli, B., Samadzadegan, F. and Reinartz, P., 2015. Fusion of hyperspectral and LiDAR data using decision template-based fuzzy multiple classifier system. International Journal of Applied Earth Observation and Geoinformation 38, pp. 309-320.

Dalponte, M., Bruzzone, L. and Gianelle, D., 2008. Fusion of hyperspectral and LiDAR remote sensing data for classification of complex forest areas. IEEE Transactions on Geoscience and Remote Sensing 46(5), pp. 1416-1427.

Debes, C., Merentitis, A., Heremans, R., Hahn, J., Frangiadakis, N., van Kasteren, T., Liao, W., Bellens, R., Pizurica, A., Gautama, S., Philips, W., Prasad, S., Du, Q. and Pacifici, F., 2014. Hyperspectral and LiDAR data fusion: Outcome of the 2013 GRSS data fusion contest. IEEE Journal of Selected Topics in Applied Earth Observations and Remote Sensing 7(6), pp. 2405-2418.

Ghamisi, P., Benediktsson, J. A. and Phinn, S., 2015. Land-cover classification using both hyperspectral and LiDAR data. International Journal of Image and Data Fusion 6(3), pp. 189-215.

Gonzalez, R. C., Woods, R. E. and Eddins, S. L., 2003. Digital Image Processing Using MATLAB. Prentice-Hall, Inc., Upper Saddle River, NJ, USA.

Gu, Y., Wang, Q., Jia, X. and Benediktsson, J., 2015. A novel MKL model of integrating LiDAR data and MSI for urban area classification. IEEE Transactions on Geoscience and Remote Sensing 53(10), pp. 5312-5326.

Holm AM, Burnside DG, M. A., 1987. The development of a system for monitoring trend in range condition in the arid shrublands of western Australia. The Australian Rangeland Journal 9(1), pp. 14-20.

Jolliffe, I., 2002. Principal Component Analysis. Wiley Online Library.

Khodadadzadeh, M., Li, J., Prasad, S. and Plaza, A., 2015. Fusion of hyperspectral and LiDAR remote sensing data using multiple feature learning. IEEE Journal of Selected Topics in Applied Earth Observations and Remote Sensing 8(6), pp. 29712983.
Luo, S., Wang, C., Xi, X., Zeng, H., Li, D., Xia, S. and Wang, P., 2016. Fusion of airborne discrete-return LiDAR and hyperspectral data for land cover classification. Remote Sensing 8(1), pp. 3-22.

Man, Q., Dong, P. and Guo, H., 2015. Pixel- and feature-level fusion of hyperspectral and LiDAR data for urban land-use classification. International Journal of Remote Sensing 36(6), pp. $1618-1644$.

Man, Q., Dong, P., Guo, H., Liu, G. and Shi, R., 2014. Light detection and ranging and hyperspectral data for estimation of forest biomass: a review. Journal of Applied Remote Sensing 8(1), pp. 081598-1-081598-21.

Matsuki, T., Yokoya, N. and Iwasaki, A., 2015. Hyperspectral tree species classification of Japanese complex mixed forest with the aid of LiDAR data. IEEE Journal of Selected Topics in Applied Earth Observations and Remote Sensing 8(5), pp. 2177-2187.

Morchhale, S., Pauca, V. P., Plemmons, R. J. and Torgersen, T. C., 2016. Classification of pixel-level fused hyperspectral and LiDAR data using deep convolutional neural networks. In: 8th Workshop on Hyperspectral Image and Signal Processing: Evolution in Remote Sensing (WHI.SPERS), Los Angeles, CA.

Pingel, T. J., Clarke, K. C. and McBride, W. A., 2013. An improved simple morphological filter for the terrain classification of airborne LiDAR data. ISPRS Journal of Photogrammetry and Remote Sensing 77, pp. $21-30$.

Priem, F. and Canters, F., 2016. Synergistic use of LiDAR and APEX hyperspectral data for high-resolution urban land cover mapping. Remote Sensing 8(10), pp. 787-809.

Wang, H. and Glennie, C., 2015. Fusion of waveform LiDAR data and hyperspectral imagery for land cover classification. ISPRS Journal of Photogrammetry and Remote Sensing 108, pp. $1-11$. 